\title{
Black's model in a negative interest rate environment, with application to OTC derivatives
}

\author{
Riccardo Bramante $^{1}$ D $\cdot$ Gimmi Dallago $^{2} \cdot$ Silvia Facchinetti $^{1}$
}

Received: 8 September 2020 / Accepted: 6 June 2021 / Published online: 2 July 2021

(c) The Author(s) 2021

\begin{abstract}
The most common application of Black's formula is interest rate derivatives pricing. Black's model, a variant of Black-Scholes option pricing model, was first introduced by Fischer Black in 1976. In recent market conditions, where global interest rates are at very low levels and in some markets are currently zero or negative, Black modelin its canonical form-fails to price interest rate options since positive interest rates are assumed in its formula. In this paper we propose a heuristic method that, without explicit assumptions about the forward rate generating process, extends the cumulative standard normal distribution domain to negative interest rates and allows Black's model to work in the conventional way. Furthermore, we provide the derivations of the so called five Greek letters that enable finance professionals to evaluate the sensitivity of an option to various parameters. Along with the description of the methodology, we present an extensive simulation study and a comparison with the Normal model which is widely used in the negative environment option pricing problems.
\end{abstract}

Keywords Black's model · Normal distribution · Negative rates · Greek letters

\section{Introduction}

After the 2008 financial crisis, some Central Banks (such as the Swiss National Bank, the European Central Bank and the Bank of Japan) took the extreme unconventional measure of setting official interest rates to zero or below in order to spur inflation and reinvigorate economic growth [see, for example, Jackson (2015)]. As a consequence, negative interest rates have become a leit-motive in financial markets, causing technical problems in many traditional formulas used to value interest rate derivatives [see

\footnotetext{
$凶$ Riccardo Bramante riccardo.bramante@unicatt.it

1 Department of Statistical Sciences, Università Cattolica del Sacro Cuore, Largo Gemelli, 1, 20123 Milan, Italy

2 Allitude S.p.A., Via Jacopo Aconcio, 9, 38122 Trento, Italy
} 
European Actuarial Association (2016); Haug (2007); Hull (2018) among others]. In fact, Black-Scholes like models usually require positive interest rates in pricing formulas and the corresponding Greeks.

Several recent papers focus on the matter of negative rate modelling on derivative pricing. Cafferata et al. (2017) study the effects played on options pricing by negative interest rates when the underlying is an equity with null dividends. Moreover, they carry out an empirical investigation to higlight the differences between estimation using some quasi-closed formulas for pricing an American option and the stochastic trinomial trees algorithm. Giribone et al. (2017) investigate the effects of negative rates on the calculation of option sensitivities by comparing the log-normal and the normal framework, both from the practical and the theoretical viewpoint. Moreover, Recchioni et al. (2017) use an adjusted Heston model (Grzelak and Oosterlee 2011) to investigate if models allowing for negative interest rates can improve option pricing and implied volatility forecasting. Finally, Friesa et al. (2017) introduce the displaced historical simulation model which handle situations of close-to-zero or negative risk variables particularly well.

Practitioners have traditionally focused on the closed-form Black's model, a variant of Black-Scholes model introduced by Fischer Black in 1976 for valuing caps, floors and swaptions (Black 1976). The mathematical framework under this model is simply unable to accept rates lower than zero, assuming positive interest rates evolve on the basis of a log-normal process. A possible solution to fix this breakdown is to include a positive adjustment to the forward value (see Sect. 2 for details).

In this paper we propose a heuristic method to solve the original Black's formula drawback by extending the cumulative standard normal distribution domain to negative interest rates. No explicit assumptions about the underlying generating process are made but, as a result, the logarithm is always well defined and the model returns a simple and stable pricing formulation on the $R_{\neq 0}$ domain of the rates thanks to which traders are able to price vanilla options using the classical log-normal framework. The use of complex numbers enables to move from the positive to the negative rates environment without creating prices discrepancies that are surely obtained when using the normal pricing structure.

The plan of the paper is as follows. In Sect. 2 some preliminary notions on BlackScholes and Black's models are introduced. In Sect. 3 we set up the methodology of our proposal. In Sect. 4 we derive the Greek letters in the context of negative interest rates, with the proof deferred to the Appendix. In Sect. 5 some evidences on options pricing in the negative domain of interest rates and a comparison with the Normal model are presented. Conclusions follow.

\section{Preliminary notions}

The Black-Scholes model is the most common option derivatives pricing framework (Black and Scholes 1973). The model assumptions are: (1) the underlying stock pays no dividend; (2) options can only be exercised upon expiration; (3) market direction cannot be predicted; (4) no commissions are charged in the transaction; (5) the volatility of the underlying and the interest rate are constant over time; (6) stock returns are 
log-normal distributed (see e.g. Shinde and Takale (2012) for details). It assumes a geometric Brownian motion $W_{t}$ for the underlying stock price $S$ such that

$$
d S=r S d_{t}+\sigma S d W_{t}
$$

under the risk-neutral measure, where $r$ is the risk-free interest rate and $\sigma$ the volatility of the stock price. Black-Scholes formulas for call and put options are as follows

$$
\begin{aligned}
& C=S N\left(d_{1}\right)-X e^{-r T} N\left(d_{2}\right) \\
& P=X e^{-r T} N\left(-d_{2}\right)-S N\left(-d_{1}\right)
\end{aligned}
$$

where

$$
\begin{aligned}
& d_{1}=\frac{\log \frac{S}{X}+\left(r+\frac{1}{2} \sigma^{2}\right) T}{\sigma \sqrt{T}} \\
& d_{2}=d_{1}-\sigma \sqrt{T}
\end{aligned}
$$

$C$ and $P$ are the call and put option price respectively, $S$ and $X$ the stock and strike price respectively, $T$ the time remaining to expiration ${ }^{1}$ and $N(\cdot)$ the cumulative probability function of a standard normal distribution.

In 1976 Black (1976) proposed a variant of Black-Scholes option pricing model that, unlike the earlier model, is useful for valuing European call or put options on future contracts. The time-evolution in (1) is revised as follows

$$
d F=\sigma F d W_{t}
$$

where the stock price $S$ of the underlying is replaced by the forward price of the rate $F$. The values of a call and a put option based on the Black formula become

$$
\begin{aligned}
& C=N_{a} e^{-r T}\left[F N\left(d_{1}\right)-X N\left(d_{2}\right)\right] \\
& P=N_{a} e^{-r T}\left[X N\left(-d_{2}\right)-F N\left(-d_{1}\right)\right]
\end{aligned}
$$

where $N_{a}$ is the notional amount and

$$
\begin{aligned}
& d_{1}=\frac{\log \frac{F}{X}+\frac{1}{2} \sigma^{2} T}{\sigma \sqrt{T}} \\
& d_{2}=d_{1}-\sigma \sqrt{T}
\end{aligned}
$$

One of the principal flaws of Black's model is that the log-normal solution does not allow rates to go below zero. This problem is empirically solved by practitioners adding a positive shift $\delta$ to the forward value in order to maintain the original analytical solutions. This approach is often called shifted Black or displaced diffusion (see e.g.

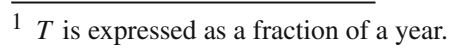


Joshi and Rebonato (2006)). This new model is similar to the Black's model, except that the forward rate $F$ is replaced with the $\delta$ shifted forward rate $(F+\delta)$

$$
d F=\sigma(F+\delta) d W_{t}
$$

Therefore, it allows negative rates, with a fixed negative lower bound defined by the amount of the $\delta$. In other words, the zero lower bound of the Black's model is shifted. One of the main criticism is that the analyst should choose a priori the shift $\delta$ to avoid negative values of the $(F+\delta)$, thus knowing the most negative value the forward rate may achieve in the future. The shifted Black's model disadvantage is that volatilities may not be quoted in the market, and then it is necessary to estimate them trough other models, like the SABR one (Hagan et al. 2002). It is a stochastic volatility model that defines the forward rate and its volatility according to the following stochastic processes:

$$
\begin{aligned}
d F & =\sigma F^{\beta} d W_{t} \\
d \sigma & =v \sigma d Z_{t}
\end{aligned}
$$

The Brownian motions driving the forward rate and the volatility are correlated each other by means of the $\rho$ coefficient:

$$
E^{Q}\left[d W_{t} d Z_{t}\right]=\rho d t
$$

Equation (12) describes the forward rate evolution with parameters $\sigma$ and $\beta$, which represents the Constant Elasticity of Variance and allows the model to switch between a lognormal model $(\beta=1)$ and a normal one $(\beta=0)$. From Eq. (13), the volatility of the forward rate $\sigma$ is itself a stochastic variable with volatility $v$.

Finally, the shifted SABR model, that introduces a shift parameter $\gamma$ in the stochastic process of the forward rate $d F=\sigma(F+\gamma)^{\beta} d W_{t}$, can be used to obtain the shifted Black volatilities when strikes are negative ${ }^{2}$ (Deloitte 2016).

\section{Methodology}

We now focus on the case of negative rates or - more generally - the case that the logarithm is undefined, e.g. the corresponding argument is negative. In this context, the value is not in the real numbers' domain and then (9) and (10) are not defined.

Considering the Black's model defined in Eqs. (7) and (8), we work to extend the domain of the standard normal distribution for values of the ratio

$$
\alpha=\frac{F}{X}<0
$$

We must therefore consider the values in the complex field.

\footnotetext{
2 Since the market gives shifted Black volatilities for some strike rate levels, the model cannot include any arbitrary size of the shift parameter. 
If we take the $\log$ of (15)

$$
\log \alpha=\log |\alpha|-i \pi
$$

Equations (9) and (10) become respectively

$$
\begin{aligned}
& d_{1}=d_{1 r}-i d_{1 c} \\
& d_{2}=d_{1 r}-\sigma \sqrt{T}-i d_{1 c}
\end{aligned}
$$

where

$$
\begin{aligned}
d_{1 r} & =\frac{\log |\alpha|+\frac{1}{2} \sigma^{2} T}{\sigma \sqrt{T}} \\
d_{1 c} & =\frac{\pi}{\sigma \sqrt{T}}
\end{aligned}
$$

The branch in $\log |\alpha|-i \pi$ ensures the continuity on $R_{\neq 0}$ of the real-valued cumulative standard normal distribution when interest rates become negative.

Now consider the standard normal distribution in the field of complex numbers

$$
G(z)=\frac{1}{\pi} e^{-z \bar{z}}
$$

where $z=a-i b, a, b \in R$ (Gallager 2008). The distribution in (21) has the well known property

$$
\int_{\mathbb{C}} G(z) d z=1
$$

Let $N_{c}(\cdot)$ be the cumulative standard normal distribution in the field of complex numbers

$$
N_{c}(z)=\int_{-\infty}^{x} \int_{-\infty}^{y} G(z) d a \cdot d b
$$

we have

$$
N_{c}(z)=\frac{1}{4}[1+\operatorname{erf}(x)][1+\operatorname{erf}(y)]
$$

where erf is the error function. Figure 1 shows an illustrative example of the cumulative standard normal distribution in the field of complex numbers.

The extension in the complex field of $N\left(d_{1}\right), N\left(d_{2}\right)$ is straightforward

$$
\begin{aligned}
& N_{c}\left(d_{1}\right)=N_{c}\left(d_{1 r}-i d_{1 c}\right) \\
& N_{c}\left(d_{2}\right)=N_{c}\left(d_{1 r}-\sigma \sqrt{T}-i d_{1 c}\right)
\end{aligned}
$$




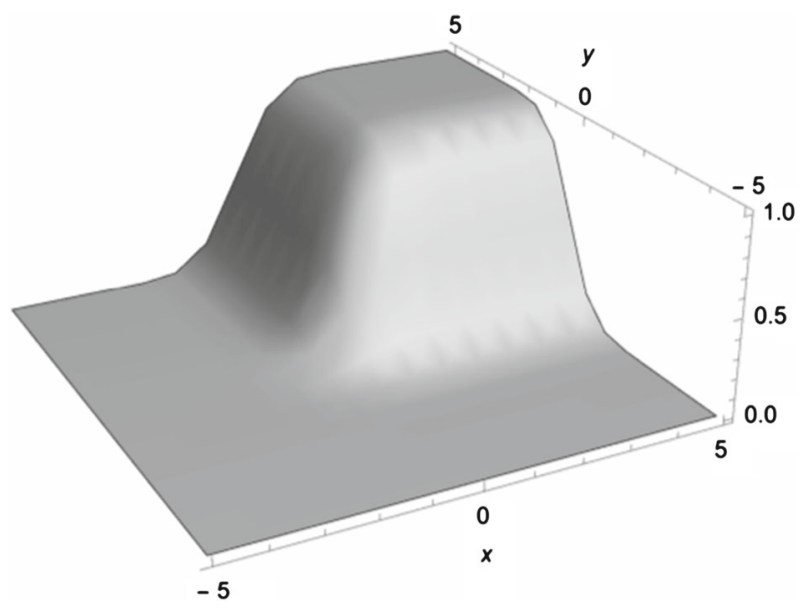

Fig. 1 Standard normal complex distribution $N_{c}(x+i y)$

Black's original formulas in (7) and (8) become ${ }^{3}$

$$
\begin{aligned}
& C=N_{a} e^{-r T}\left[F N_{c}\left(d_{1}\right)-X N_{c}\left(d_{2}\right)\right] \\
& P=N_{a} e^{-r T}\left[X N_{c}\left(-d_{2}\right)-F N_{c}\left(-d_{1}\right)\right]
\end{aligned}
$$

where

$$
\begin{aligned}
N_{c}\left(d_{1 r}-i d_{1 c}\right) & =\frac{1}{4}\left[1+\operatorname{erf}\left(d_{1 r}\right)\right]\left[1-\operatorname{erf}\left(d_{1 c}\right)\right] \\
N_{c}\left(d_{1 r}-\sigma \sqrt{T}-i d_{1 c}\right) & =\frac{1}{4}\left[1+\operatorname{erf}\left(d_{1 r}-\sigma \sqrt{T}\right)\right]\left[1-\operatorname{erf}\left(d_{1 c}\right)\right]
\end{aligned}
$$

Equations (27) and (28) are equivalent to (7) and (8) when the real numbers domain is considered. In fact, if we extend the assumptions on $\alpha$ considering only $\alpha>0$ then the imaginary part of $\log \alpha$ is real. The cumulative standard normal distribution is as follows

$$
N(x)=\frac{1}{2}\left[1+\operatorname{erf}\left(\frac{x}{\sqrt{2}}\right)\right]
$$

and is equal to $N(x+i 0)$ since $\operatorname{erf}(0)=0$.

From (31) we have

$$
N^{\prime}(x)=\frac{1}{\sqrt{2 \pi}} e^{-x^{2}}
$$

\footnotetext{
3 All the proposed pricing formulas are referred to a single caplet or floorlet.
} 
Complex derivation follows from (21)

$$
N_{c}^{\prime}(z)=\frac{1}{\pi} e^{-z \bar{z}}
$$

\section{Greek letters}

The five Greek letters (or simply the Greeks) represent the sensitivity of the option price with respect to the parameters that define the model of the underlying asset. Each letter measures a different risk dimension in an option position [(see Giribone et al. (2017), Hull (2018)] and is evaluated as an appropriate partial derivatives of the option price. In this section we derive the mathematical formulas of the first-order (delta, theta, vega and omega) and the second-order (gamma) Greeks both for a single caplet and floorlet option ${ }^{4}$. The complete proofs are deferred to the Appendix.

Delta $(\Delta)$ measures the sensitivity of the option relative to the underlying. It is the derivative of the option price with respect to the forward price $F$

$$
\begin{aligned}
\Delta_{(\text {Caplet })} & =\frac{\partial C}{\partial F}=N_{a} e^{-r T} N_{c}\left(d_{1}\right) \\
\Delta_{(\text {Floorlet })} & =\frac{\partial P}{\partial F}=N_{a} e^{-r T}\left[N_{c}\left(d_{1}\right)-1\right]
\end{aligned}
$$

Theta $(\Theta)$ represents the price sensitivity to the passage of time. It is the derivative of the option value with respect to the time $T$

$$
\begin{aligned}
\Theta_{(\text {Caplet })} & =\frac{\partial C}{\partial T}=r C-\frac{N_{a} F \sigma}{2 \sqrt{T}} e^{-r T} N_{c}^{\prime}\left(d_{1}\right) \\
\Theta_{(\text {Floorlet })} & =\frac{\partial P}{\partial T}=r P-\frac{N_{a} F \sigma}{2 \sqrt{T}} e^{-r T} N_{c}^{\prime}\left(d_{1}\right)
\end{aligned}
$$

Vega $(v)$ measures the sensitivity to volatility. It is the derivative of the option price with respect to the volatility $\sigma$ of the underlying asset ${ }^{5}$

$$
v_{(\text {Caplet })}=\frac{\partial C}{\partial \sigma}=F N_{a} e^{-r T} N_{c}^{\prime}\left(d_{1}\right) \sqrt{T}
$$

\footnotetext{
${ }^{4}$ In addition to Greeks, the sensitivity of the option price with respect to the strike rate $X$ is usually considered. In detail, for caplet options

$$
\frac{\partial C}{\partial X}=-N_{a} e^{-r T} N\left(d_{2}\right)
$$

whereas for floorlet options

$$
\frac{\partial P}{\partial X}=N_{a} e^{-r T} N\left(-d_{2}\right)
$$

5 Like in the standard Black's framework $v_{(\text {Caplet })}=v_{(\text {Floorlet })}$.
} 


$$
\nu_{(\text {Floorlet })}=\frac{\partial P}{\partial \sigma}=F N_{a} e^{-r T} N_{c}^{\prime}\left(d_{1}\right) \sqrt{T}
$$

Rho $(\rho)$ measures the sensitivity to the interest rate. It is the derivative of the option value with respect to the risk free interest rate $r$

$$
\begin{gathered}
\rho_{(\text {Caplet })}=\frac{\partial C}{\partial r}=-T C \\
\rho_{(\text {Floorlet })}=\frac{\partial P}{\partial r}=-T P
\end{gathered}
$$

Gamma $(\Gamma)$ represents the rate of change between $\Delta$ and the underlying asset price. It is the second-order derivative of the option price with respect to the forward price $F^{6}$

$$
\begin{gathered}
\Gamma_{(\text {Caplet })}=\frac{\partial^{2} C}{\partial F^{2}}=\frac{\partial \Delta_{(\text {Caplet })}}{\partial F}=\frac{N_{a}}{F \sigma \sqrt{T}} e^{-r T} N_{c}^{\prime}\left(d_{1}\right) \\
\Gamma_{(\text {Floorlet })}=\frac{\partial^{2} P}{\partial F^{2}}=\frac{\partial \Delta_{(\text {Floorlet })}}{\partial F}=\frac{N_{a}}{F \sigma \sqrt{T}} e^{-r T} N_{c}^{\prime}\left(d_{1}\right)
\end{gathered}
$$

\section{Simulation study}

In this section some evidence on interest rates options pricing in the negative domain is reported. The application context is that of interest rate Over The Counter (OTC) derivatives or floating rate loans and bonds with embedded cap or floor options. Being the result of a private transaction between the buyer and the seller or parts of a financial instrument that includes a nonderivative host contract, these contracts are customized in their characteristics (among all expiration date, type of exercise, number of units of the underlying asset) and there is no secondary market [(see e.g. Fabozzi et al. (2003)]. For these reasons a great need of statistical and mathematical methods to determine the option price and measure the risk of the corresponding positions is necessary.

In order to consider different scenarios, we run an extensive simulation study by evaluating options pricing, along with the corresponding Greeks, by varying the main parameters: forward rate $F$, strike price $X$, risk-free interest rate $r$, volatility $\sigma$, time to maturity $T$ (in months). The list of the examined ranges is reported in Table $1^{7}$.

A Mathematica ${ }^{\mathrm{TM}}$ Package ${ }^{8}$ has been developed for options pricing and simulation in the proposed framework. Our program evaluates Eq. (24) in the field of complex numbers (e.g. when interest rates are negative), employing the usual standard normal distribution function when interest rates are positive ${ }^{9}$. Considering each possible

\footnotetext{
${ }^{6}$ Like in the standard Black's framework $\Gamma_{(\text {Caplet })}=\Gamma_{(\text {Floorlet })}$.

7 All the ranges reported in Table 1, leaving out the $T$ parameter, are expressed as percentages.

8 The package is available upon request.

9 The program combines the error function erf and the chop one that replaces approximate real numbers in expr that are close to zero by the corresponding exact.
} 
Table 1 List of examined ranges

\begin{tabular}{llll}
\hline Parameters & Min & Max & Step \\
\hline$F$ & -1.5 & 1.5 & 0.1 \\
$X$ & -1.5 & 1.5 & 0.1 \\
$r$ & -1.0 & 1.0 & 0.1 \\
$\sigma$ & 0.1 & 0.4 & 0.05 \\
$T$ & 1 & 12 & 1 \\
\hline
\end{tabular}

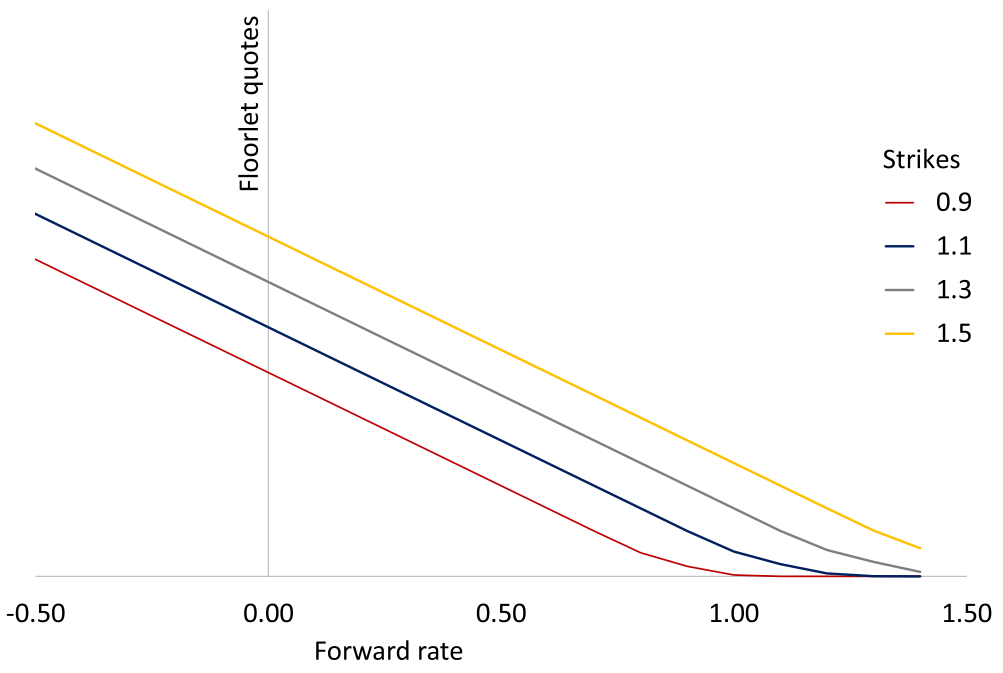

Fig. 2 Floorlet options: $r=0, \sigma=0.1, T=9$ months

combination of the parameters reported in Table 1, we get about 2 million of pricing values for the two type of options (caplet/floorlet).

To illustrate the strength of the proposed option pricing formula, here below we report some results of our simulation analysis.

Figure 2 shows the theoretical price of a floorlet option $(r=0, \sigma=0.1, T=9$ months) with respect to the forward rate, for some of the considered range of strike prices. The option pricing function $P$ is continuous on the whole $R_{\neq 0}$ domain of interest rates and is capable to ensure continuity around zero values of $F$.

As for Greeks, all the patterns with respect to the option parameters resemble the ones of the original Black's model. Figure 3 illustrates, as an example, Delta behaviour at various levels of the forward rate for some of the considered range of volatilities in a floorlet option case ( $X=1.1, r=0, T=9$ months), where the increase in Deltas with respect to volatilities is the one expected.

Moreover, pricing values obtained with the proposed framework and the corresponding ones of the Normal and Shifted Black models are compared. For the sake of simplicity and to avoid redundancy, only the Normal model results are reported 


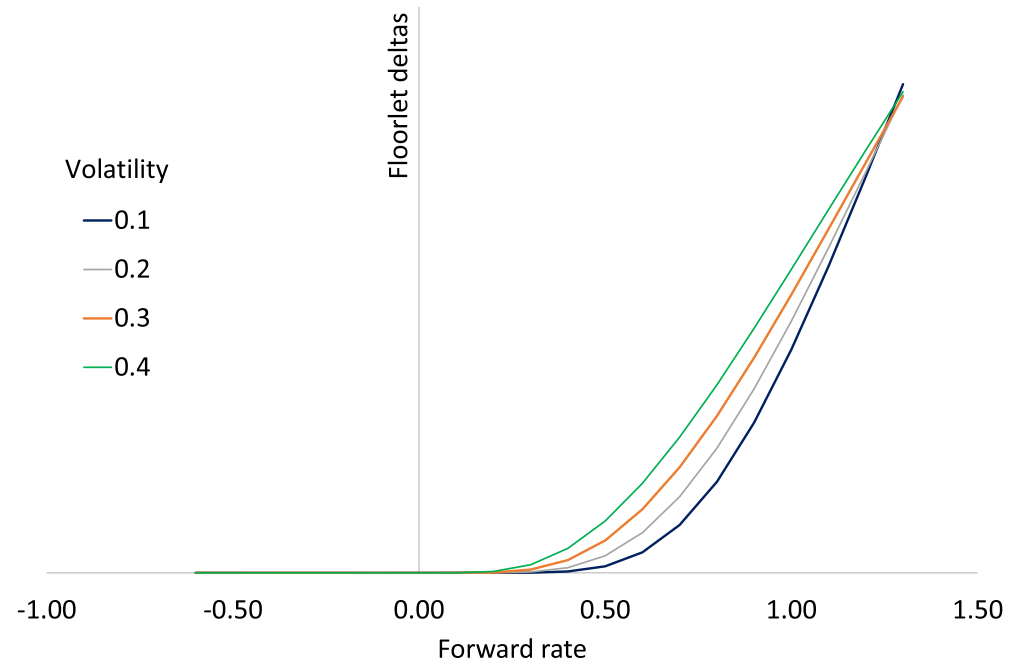

Fig. 3 Floorlet deltas: $X=1.1, r=0, T=9$ months

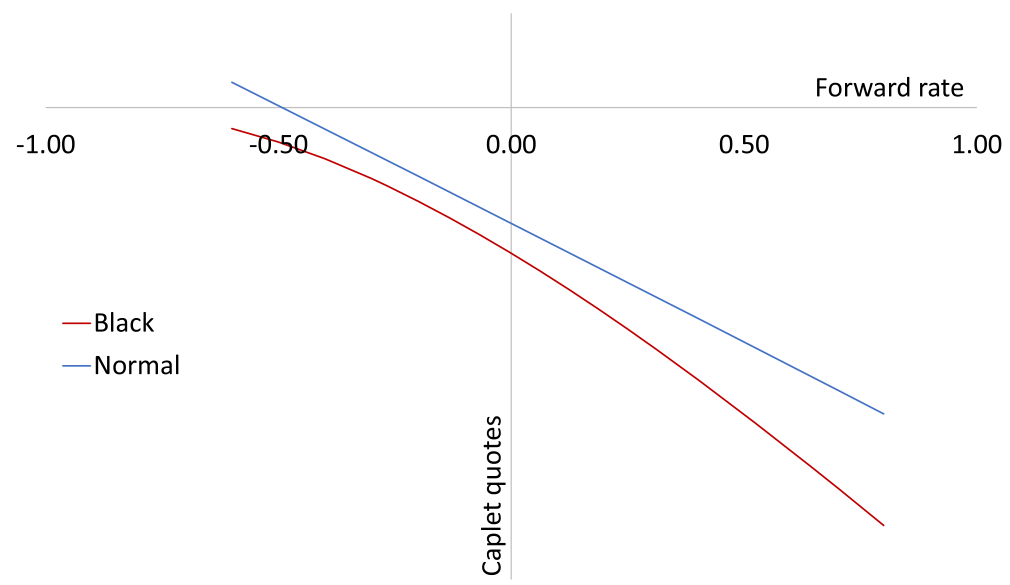

Fig. 4 Black's and Normal models comparison for a caplet option: $X=1.3, r=-0.6, \sigma=0.2, T=11$ months

hereinafter ${ }^{10}$. In Figs. 4 and 5 pricing results by varying the forward rate are showed: pricing values are similar over the whole domain of the forward rate with the main deviations that are reported when the extremes of $F$ are approached ${ }^{11}$. Similar results are obtained for all the considered contracts.

Finally, in order to check if the two considered models are overall aligned in terms of pricing values, we have performed a standard correlation analysis for different levels

\footnotetext{
10 The Normal model is considered a robust and reliable model and is based on the Bachelier pricing framework for pricing the vanilla derivatives [see e.g. Jamshidian (1997); Terakado (2019)].

11 As for the floorlet option, due to the type of contract, deviations are only in the $F$ negative domain since the models' pricing converges to zero when $F$ moves away from the hypothesized strike price.
} 


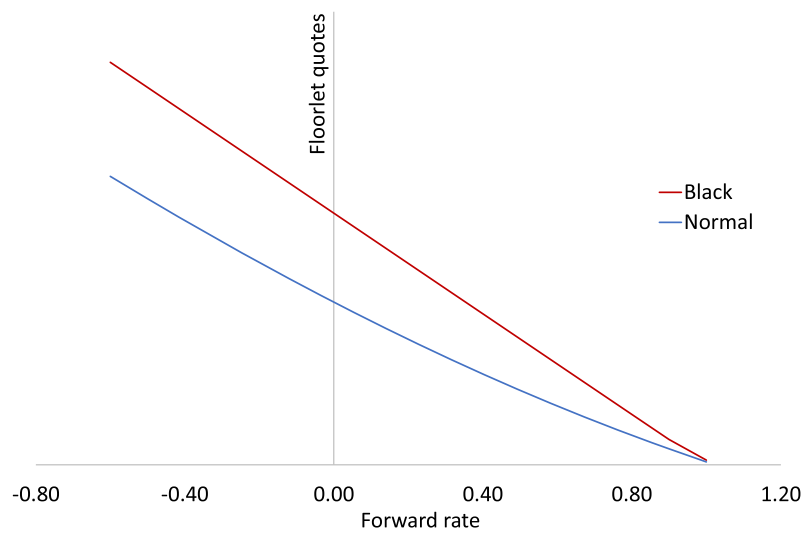

Fig. 5 Black's and Normal models comparison for a floorlet option: $X=1.5, r=0.5, \sigma=0.1, T=1$ month

of the parameters strike price, volatility, risk-free interest rate and time to maturity. Globally speaking, the average correlation is about $97 \%$ when floorlet options are considered, whereas about $95 \%$ when caplet contracts are examined thus confirming that the two models are aligned from the point of view of the pricing values provided.

When the analysis is partitioned into all the parameters excluding the volatility, average correlations remain close to the global ones, irrespective of the contract type. On the other hand, we observe a decrease in floorlet options average correlation as volatility increases (see Fig. 6); anyway, the lower average correlation value is near to $90 \%$ when $\sigma=40 \%$, still ensuring a quite good match between the two models.

\section{Concluding remarks}

This paper contributes to the debate on the use of Black's model when interest rates follow into negative territory. Our paper shows that an intuitive strategy for using the original formula in a negative-rate environment is to restate the cumulative standard normal distribution domain in the complex field. This is achieved by means of a specific branch of $\log (F / X)$ when passing through the complex domain, without creating option price discrepancies that can be obtained when using the normal pricing framework. Based on this framework new Greeks are introduced.

Finally, a comparison of our proposal with the Normal model shows that the prices are similar over the whole domain of the forward rate, and a standard correlation analysis confirms that the two models are aligned from the point of view of the pricing values provided. Thus, the proposed methodology represents a simple and valid alternative to the main solutions adopted by the most widespread pricing services.

Further research is continuing on the evaluation of the model developed in this paper. Specifically, our work is primarily focused on how the forward price is determined 


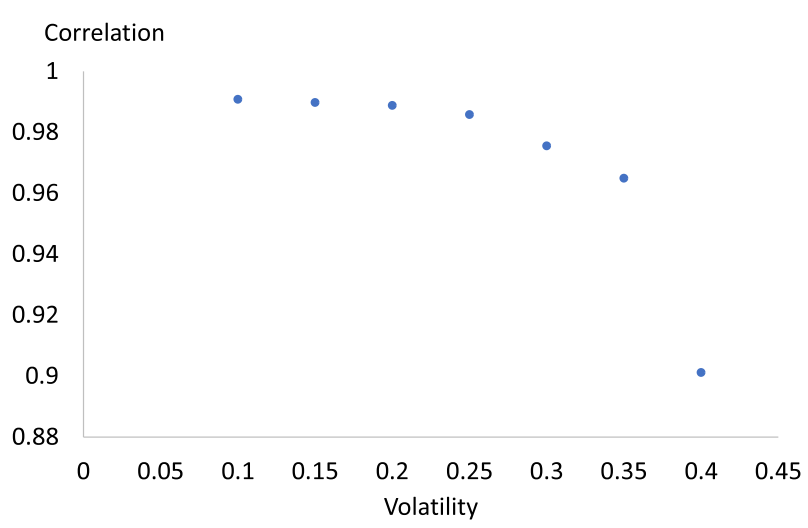

\begin{tabular}{c|c} 
Volatility & Correlation \\
\hline 0.10 & 0.99081 \\
0.15 & 0.98980 \\
0.20 & 0.98882 \\
0.25 & 0.98583 \\
0.30 & 0.97556 \\
0.35 & 0.96495 \\
0.40 & 0.90120
\end{tabular}

Fig. 6 Floorlet average correlations by volatility

investigating the stochastic model for the spot price dynamics when the Gaussian distribution in the field of complex numbers is used.

Funding Open access funding provided by Universitá Cattolica del Sacro Cuore within the CRUI-CARE Agreement.

Open Access This article is licensed under a Creative Commons Attribution 4.0 International License, which permits use, sharing, adaptation, distribution and reproduction in any medium or format, as long as you give appropriate credit to the original author(s) and the source, provide a link to the Creative Commons licence, and indicate if changes were made. The images or other third party material in this article are included in the article's Creative Commons licence, unless indicated otherwise in a credit line to the material. If material is not included in the article's Creative Commons licence and your intended use is not permitted by statutory regulation or exceeds the permitted use, you will need to obtain permission directly from the copyright holder. To view a copy of this licence, visit http://creativecommons.org/licenses/by/4.0/.

\section{Appendix: derivations of Greek letters}

All the proofs of Greek letters for both caplet and floorlet options in our proposal are provided. 


\subsection{Derivation of equations (34) and (35)}

$$
\begin{aligned}
\Delta_{(\text {Caplet })} & =\frac{\partial C}{\partial F} \\
& =N_{a} e^{-r T}\left[N_{c}\left(d_{1}\right)+F N_{c}^{\prime}\left(d_{1}\right) \frac{\partial d_{1}}{\partial F}-X N_{c}^{\prime}\left(d_{2}\right) \frac{\partial d_{2}}{\partial F}\right] \\
& =N_{a} e^{-r T}\left[N_{c}\left(d_{1}\right)+\frac{N_{c}^{\prime}\left(d_{1}\right)}{\sigma \sqrt{T}}-N_{c}^{\prime}\left(d_{2}\right) \frac{N_{c}^{\prime}\left(d_{1}\right)}{\sigma \sqrt{T}}\right] \\
& =N_{a} e^{-r T} N_{c}\left(d_{1}\right) \\
\Delta_{(\text {Floorlet })} & =\frac{\partial P}{\partial F} \\
& =N_{a} e^{-r T}\left[-N_{c}\left(-d_{1}\right)-F \frac{\partial}{\partial d_{1}}\left(1-N_{c}\left(d_{1}\right)\right) \frac{\partial d_{1}}{\partial F}+X \frac{\partial}{\partial d_{2}}\left(1-N_{c}\left(d_{2}\right)\right) \frac{\partial d_{2}}{\partial F}\right] \\
& =N_{a} e^{-r T}\left[N_{c}\left(d_{1}\right)-1+\frac{N_{c}^{\prime}\left(d_{1}\right)}{\sigma \sqrt{t}}-X N_{c}^{\prime}\left(d_{2}\right) \frac{1}{F \sigma \sqrt{T}}\right] \\
& =N_{a} e^{-r T}\left[N_{c}\left(d_{1}\right)-1\right]
\end{aligned}
$$

where

$$
\frac{\partial d_{1}}{\partial F}=\frac{\partial d_{2}}{\partial F}=\frac{1}{F} \frac{1}{\sigma \sqrt{T}}
$$

\subsection{Derivation of equations (36) and (37)}

$$
\begin{aligned}
\Theta_{(\text {Caplet })} & =\frac{\partial C}{\partial T} \\
& =N_{a} r e^{-r T}\left[F N_{c}\left(d_{1}\right)-X N_{c}\left(d_{2}\right)\right] \\
& -N_{a} e^{-r T}\left[F N_{c}^{\prime}\left(d_{1}\right) \frac{\partial d_{1}}{\partial T}-X N_{c}^{\prime}\left(d_{2}\right) \frac{\partial d_{2}}{\partial T}\right] \\
& =r C-\frac{N_{a} F \sigma}{2 \sqrt{T}} e^{-r T} N_{c}^{\prime}\left(d_{1}\right) \\
\Theta_{(\text {Floorlet })} & =\frac{\partial P}{\partial T} \\
& =N_{a} r e^{-r T}\left[X N_{c}\left(-d_{2}\right)-F N_{c}\left(-d_{1}\right)\right] \\
& -N_{a} e^{-r T}\left[X \frac{\partial}{\partial d_{2}}\left(1-N_{c}\left(d_{2}\right)\right) \frac{\partial d_{2}}{\partial T}-F \frac{\partial}{\partial d_{1}}\left(1-N_{c}\left(d_{1}\right)\right) \frac{\partial d_{1}}{\partial T}\right] \\
& =r P-\frac{N_{a} F \sigma}{2 \sqrt{T}} e^{-r T} N_{c}^{\prime}\left(d_{1}\right)
\end{aligned}
$$

where

$$
\frac{\partial d_{1}}{\partial T}=\frac{\sigma}{2 \sqrt{T}}-\frac{d_{1}}{2 T} \quad \frac{\partial d_{2}}{\partial T}=-\frac{d_{1}}{2 T}
$$




\subsection{Derivation of equations (38) and (39)}

$$
\begin{aligned}
\nu_{(\text {Caplet })} & =\frac{\partial C}{\partial \sigma} \\
& =N_{a} e^{-r T}\left[F N_{c}^{\prime}\left(d_{1}\right) \frac{\partial d_{1}}{\partial \sigma}-X N_{c}^{\prime}\left(d_{2}\right) \frac{\partial d_{2}}{\partial \sigma}\right] \\
& =N_{a} e^{-r T}\left[F N_{c}^{\prime}\left(d_{1}\right)\left(\sqrt{T}-\frac{d_{1}}{\sigma}\right)+F N_{c}^{\prime}\left(d_{1}\right) \frac{d_{1}}{\sigma}\right] \\
& =F N_{a} e^{-r T} N_{c}^{\prime}\left(d_{1}\right) \sqrt{T} \\
\nu_{(\text {Floorlet })} & =\frac{\partial P}{\partial \sigma} \\
& =N_{a} e^{-r T}\left[X \frac{\partial}{\partial d_{2}}\left(1-N_{c}\left(d_{2}\right)\right) \frac{\partial d_{2}}{\partial \sigma}-F \frac{\partial}{\partial d_{1}}\left(1-N_{c}\left(d_{1}\right)\right) \frac{\partial d_{1}}{\partial \sigma}\right] \\
& =N_{a} e^{-r T}\left[X N_{c}^{\prime}\left(d_{2}\right) \frac{d_{1}}{\sigma}+F N_{c}^{\prime}\left(d_{1}\right)\left(\sqrt{T}-\frac{d_{1}}{\sigma}\right)\right] \\
& =F N_{a} e^{-r T} N_{c}^{\prime}\left(d_{1}\right) \sqrt{T}
\end{aligned}
$$

where

$$
\frac{\partial d_{1}}{\partial \sigma}=\sqrt{T}-\frac{d_{1}}{\sigma} \quad \frac{\partial d_{2}}{\partial \sigma}=-\frac{d_{1}}{\sigma}
$$

\subsection{Derivation of equations (40) and (41)}

$$
\begin{aligned}
\rho_{(\text {Caplet })} & =\frac{\partial C}{\partial r} \\
& =-N_{a} T e^{-r T}\left[F N_{c}\left(d_{1}\right)-X N_{c}\left(d_{2}\right)\right] \\
& =-T C \\
\rho_{(\text {Floorlet })} & =\frac{\partial P}{\partial r} \\
& =-N_{a} T e^{-r T}\left[X N_{c}\left(-d_{2}\right)-F N_{c}\left(-d_{1}\right)\right] \\
& =-T P
\end{aligned}
$$

where

$$
\frac{\partial d_{1}}{\partial r}=\frac{\partial d_{2}}{\partial r}=0
$$

\subsection{Derivation of equations (42) and (43)}

$$
\Gamma_{(\text {Caplet })}=\frac{\partial^{2} C}{\partial F^{2}}=\frac{\partial \Delta_{(\text {Caplet })}}{\partial F}
$$




$$
\begin{aligned}
& =N_{a} e^{-r T} \frac{\partial}{\partial d_{1}} N_{c}\left(d_{1}\right) \frac{\partial d_{1}}{\partial F} \\
& =\frac{N_{a}}{F \sigma \sqrt{T}} e^{-r T} N_{c}^{\prime}\left(d_{1}\right) \\
\Gamma_{(\text {Floorlet })} & =\frac{\partial^{2} P}{\partial F^{2}}=\frac{\partial \Delta_{(\text {Floorlet })}}{\partial F} \\
& =N_{a} e^{-r T} \frac{\partial}{\partial d_{1}} N_{c}\left(d_{1}\right) \frac{\partial d_{1}}{\partial F} \\
& =\frac{N_{a}}{F \sigma \sqrt{T}} e^{-r T} N_{c}^{\prime}\left(d_{1}\right)
\end{aligned}
$$

\section{References}

Black F (1976) The pricing of commodity contracts. J Financ Econ 3:167-179

Black F, Scholes M (1973) The pricing of options and corporate liabilities. J Political Econ 81:637-654

Cafferata A, Giribone PG, Resta M (2017) The effects of negative nominal rates on the pricing of american calls: some theoretical and numerical insights. Modern Econ 8:878-887

Deloitte (2016) Interest rate derivatives in the negative-rate environment: Pricing with a shift. Available at: https://www2.deloitte.com/content/dam/Deloitte/global/Documents/Financial-Services/gxbe-aers-fsi-pricing-with-negative-rates.pdf

European Actuarial Association (2016) Negative interest rates and their technical consequences. Available at: http://actuary.eu/documents/AAE-negative-interest-rates_FINAL-161216.pdf

Fabozzi FJ, Mann SV, Choudhry M (2003) Measuring and controlling interest rate and credit risk, 2nd edn. Wiley, Hoboken

Friesa CP, Nigburb T, Seegerc N (2017) Displaced relative changes in historical simulation: application to risk measures of interest rates with phases of negative rates. J Empir Financ 42:175-198

Gallager R (2008) Circularly-symmetric gaussian random vectors. Available at: http://www.rle.mit.edu/ rgallager/documents/CircSymGauss.pdf

Giribone PG, Ligato S, Mulas M (2017) The effects of negative interest rates on the estimation of option sensitivities: the impact of switching from a log-normal to a normal model. Int J Financ Eng 4:1-42

Grzelak LA, Oosterlee CW (2011) On the Heston model withstochastic interest rates. SIAM J Financ Math 2:255-286

Hagan PS, Kumar D, Lesniewski AS, Woodward DE (2002) Managing smile risk. Wilmott Mag 1:84-108 Haug EG (2007) The complete guide to option pricing formulas, 2nd edn. McGraw-Hill, New York City

Hull JC (2018) Options, futures and other derivates, 10th edn. Pearson, London

Jackson H (2015) The international experience with negative policy rates. Bank of Canada Staff Discussion Paper 2015-13

Jamshidian F (1997) LIBOR and swap market models and measures. Financ Stochast 1:293-330

Joshi M, Rebonato R (2006) A stochastic-volatility, displaced-diffusion extension of the LIBOR market model. Quant Financ 3:458-469

Recchioni MC, Sun Y, Tedeschi G (2017) Can negative interest rates really affect option pricing? Empirical evidence from an explicitly solvable stochastic volatility model. Quanti Financ 17:1257-1275

Shinde AS, Takale KC (2012) Study of Black-Scholes model and its applications. Proc Eng 38:270-279

Terakado S (2019) On the option pricing formula based on the bachelier model. Available at. https://doi. org/10.2139/ssrn.3428994

Publisher's Note Springer Nature remains neutral with regard to jurisdictional claims in published maps and institutional affiliations. 